Final Report for Period: 06/2004 - 05/2005

Submitted on: 07/13/2005

Principal Investigator: Roberts, Richard J.

Award ID: 0434632

Organization: Amer Soc For Microbiology

Title:

Colloquium: An Experimental Approach to Genome Annotation to be held July 19-20, 2004 in Washington, DC

\title{
Senior Personnel
}

\section{Project Participants}

Name: Roberts, Richard

Worked for more than 160 Hours: Yes

Contribution to Project:

\section{Post-doc}

\section{Graduate Student}

\section{Undergraduate Student}

\section{Technician, Programmer}

\section{Other Participant}

\section{Research Experience for Undergraduates}

\section{Organizational Partners}

\section{Other Collaborators or Contacts}

One of the key features of this colloquium was to deliberate the potential funding schemes. Representatives from the main U.S. funding agencies were invited to attend the colloquium. There were representatives from the following agencies: NASA; NOAA; OSTP; NSF; DOE; $\mathrm{NIH}$; and USDA.

\section{$\underline{\text { Activities and Findings }}$}

\section{Research and Education Activities:}

The American Academy of Microbiology convened a colloquium of 33 scientific experts and 14 observers from various federal agencies on July 19-20, 2004, in Washington, DC, to discuss the problems faced in annotating the many complete microbial genome sequences that are now known. There are severe problems with the current level of annotation of these genomes, and this is hampering their full utilization. Many of these genomes are known human pathogens. A more complete knowledge of their biology, which will be revealed by discerning the function of their genes, will be of value to clinicians (who aim to tackle the diseases they cause), drug finders (who aim to find cures), and bioterrorism experts (who are concerned both with diagnostics and with effective countermeasures to these pathogens, should they be used for terrorist attacks).

The result of this colloquium is the publication, 'An Experimental Approach to Genome Annotation.' It has been widely publicized and distributed, both electronically and in print, to the scientific community and general public.

\section{Findings:}

Recent advances in DNA sequencing have produced a spectacular amount of new data; literally hundreds of thousands of sequenced 
prokaryotic genes now await annotation. These genes can be enumerated, compared, and grouped by sequence similarity into families, yet an understanding of their biochemical functions is lacking. Genomics provides that rare opportunity in science where the boundaries of current knowledge can be clearly defined.

Colloquium participants agreed that accurate and complete annotation is vital to making full use of genomic data. There are great deficiencies in currently available annotation sources, and there are few sources of dedicated funding for experimental approaches to annotation.

It was recommended that a new initiative be undertaken that would synergistically combine computational methodologies for functional prediction with a systematic experimental approach to test those predictions. It would also broaden the foundational set of experimentally determined gene functions by finding missing genes for known enzymatic functions. Such a program would both increase experimental knowledge and spur further accuracy in bioinformatics prediction leading to repeated cycles of validation and prediction.

Four areas of concern in genomic annotation were identified:

(1) As many as $40 \%$ of all predicted genes in completed prokaryotic genomes have no functional annotation.

(2) Many genes have a predicted function, but that prediction has not been experimentally validated.

(3) As many as $5-10 \%$ of predicted gene functions may

be incorrect.

(4) Many known enzymes have no corresponding genes identified in the sequence databases.

As part of the proposed initiative, a new resource focused on annotation should be developed. The central component is a database containing:

-Predictions regarding the functions of genes of unknown function, deposited by bioinformaticians, based on computationally inferred clues, which will serve as a starting point for experimental investigations.

-The results, positive or negative, of those experimental

investigations, which in many cases will establish new gene annotations backed by rigorous experimental work.

-A prioritized list of sequenced genes for which no functional information is currently available.

-A list of biochemically-characterized functions for which no gene has yet been assigned (referred to as orphan functions).

-Data on previously characterized proteins currently in the public databases.

The basic design of the database was discussed, and recommendations for hosting, administration, and management of the database were put forth.

\section{Training and Development:}

The colloquium brought together bioinformaticians and experimental biologists to discuss how a synergy between the two groups might be established. The participants were divided by into small working groups; each group had a mix of bioinformaticians and experimental biologists. The groups discussed the same issues and independently developed a consensus response to the issues which represented each participant's expertise and experience.

This interdisciplinary approach was a very stimulating exchange which allowed the participants to learn how the other participants are handling these issues.

A key feature of this colloquium was to deliberate potential funding schemes. Numerous representatives from the main U.S. funding agencies were present as observers. It was of great value for the participants and the observers to hear the discussion of the problems faced in genome annotation.

\section{Outreach Activities:}

A professional science writer, working with the steering committee, drafted the report, summarizing the discussions and recommendations of the working groups from the colloquium. After extensive review by colloquium participants and further peer review by the Board of the American Academy of Microbiology, the report was then published, both in print and electronic formats. A specific outreach plan was developed and implemented, including: 
A press release announcing the report was sent to the New York Times and Washington Post as well as general scientific publications, 21st Century Science \& Technology; AIG Journal; American Scientist;

Biochemistry; Biology Digest; BioPharm; Bioscience; Biotechniques;

Engineering \& Science Review; Engineering and Science; Health Policy and Biomedical Research-The Blue Sheet; Issues in Science and Technology; Journal of Cell Biology; Nature BioTechnology; New Scientist; Science; Science Daily Magazine; Science News; Science Scope; Science Watch; and The Scientist.

The report was posted in the 'What's New' section of the American Society for Microbiology (ASM) website,www.asm.org, and announced to the ASM members in the following scientific divisions: microbiology education; genetics and molecular biology; general microbiology; evolutionary and genomic microbiology; and molecular, cellular, and general eukaryotes.

A specific webpage (http://www.asm.org/Academy/index.asp?bid=32664) was also developed, within the American Academy of Microbiology's (AAM) section of the ASM website containing two downloadable PDF versions of the report. This url was added to the yahoo and google search engines.

Copies of the report were provided to the leadership of ASM and the AAM,AAM Fellows, and colloquium participants and observers.

The report was announced in the February 2005 ASM international listserv which is distributed to 2,500 subscribers.

The report was also posted in the Weekly News Digest of ASM news and to the American Academy of Arts and Sciences Eurekalert.

\section{Journal Publications}

\section{Books or Other One-time Publications}

Richard J. Roberts, Peter Karp, Simon Kasif, Stuart Linn, and Merry R. Buckley, "An Experimental Approach to Genome Annotation", (2004). Book, Published

Bibliography: Washington, DC: American Society for Microbiology

\section{Web/Internet Site}

\section{URL(s):}

http://www.asm.org/academy/index.asp?bid=32664

\section{Description:}

This site has a copy of the colloquium report that is the product of this award.

\section{Other Specific Products}

\section{Contributions}

\section{Contributions within Discipline:}

Considering the importance of genome annotation to full exploitation of sequence information, progress in experimental annotation has been slow, largely due to a lack of available funding for experimental annotation approaches. It is recommended that an annotation initiative be undertaken to catalyze and coordinate funding for experimental approaches to genome annotation.

-Given the current lack of a reliable source of functional annotation data, a central data resource should be established. It should incorporate: a database of peer-reviewed, experimentally verified gene annotations; a catalog of the genes that have yet to be annotated which users can sort by gene family, species, priority, etc.,; a catalog of the functions for which a gene remains to be found; and all available experimental information relevant to function. 
-Priority in designating funding for annotation through the initiative should be placed first on prokaryotic genomes. In selecting among prokaryotic genes, emphasis should be placed on those gene products that belong to large protein families, since knowledge of these genes is most likely to impart an understanding of the biology of many diverse systems. In this way, a small investment of experimental work and funding can lead to big rewards in understanding many species.

-Progress in functional annotation could be enhanced to some extent by developing mechanisms and information systems that encourage collaboration between bioinformaticians and experimentalists. This would allow experimental scientists to quickly access and test the predictions made using informatics tools, while providing bioinformaticians access to experts on the function of particular genes and enzyme systems.

-In efforts to identify the gene encoding a given product, funding priority should be given to those proteins or RNAs that have been purified and characterized, as simple sequencing would then lead to

identification of the gene.

-The design of the database should remain open to the input of researchers who choose to submit proposals for constructing and maintaining it. However, it must support the maintenance and update of working hypotheses about gene function.

-To avoid conflicts with the interests of potential contributors, the database should be hosted by an unbiased organization without a vested interest in the content of the data.

-The collaborative contributions of experimentalists and bioinformaticians should be encouraged through the requests for applications announced by the database coordinators.

-A variety of funding types will be needed, including small awards to support students who might work in an experienced investigator's laboratory for a short period of time.

-The resources produced by the initiative should be made public and be freely available to the global scientific community.

\section{Contributions to Other Disciplines:}

The potential impact of the proposed initiative is difficult to overstate since it would affect all aspects of biology. The participants feel that this project is essential to enable the next step in moving genomic science forward from accumulating a large depository of sequences towards achieving a true understanding of

the basic elements of prokaryotic biology. Without a forward-

looking initiative like the one proposed here, the functional data needed to propel systems biology forward will not be available, and those trying to understand the complex interactions of genes and their

products in living cells will continue to work with many components of unknown function. In addition, elucidating the enzymatic functions essential for prokaryotic life will impact our understanding of eukaryotic organisms, which possess many of these same genes. This initiative will also foster closer collaborations between experimental and computational scientists and help to reinstate the importance of biochemical research.

Finally, although much of the project will focus on traditional

biochemistry, the initiative can be expected to stimulate new advances in functional screening, new functional genomic technologies such as phenotype arrays, and significant industrial and commercial opportunities in the form of new targets for both medical and industrial applications of prokaryotic biology.

\section{Contributions to Human Resource Development:}

\section{Contributions to Resources for Research and Education:}

\section{Contributions Beyond Science and Engineering:}

The annotation initiative proposed will extend the boundaries of current knowledge and will likely lead to new applications and new progress in healthcare, biodefense, energy, the environment, and agriculture. This research could also impact many commercial enterprises, such as the chemical, food and dairy industries. 


\section{Categories for which nothing is reported:}

Organizational Partners

Any Journal

Any Product

Contributions: To Any Human Resource Development

Contributions: To Any Resources for Research and Education 